\title{
Thermomechanical Properties and Glass Dynamics of Polymer- Tethered Colloidal Particles and Films
}

Yu Cang, ${ }^{\dagger}$ Anna N. Reuss, ${ }^{\dagger}$ Jaejun Lee, ${ }^{\ddagger}$ Jiajun Yan, ${ }^{\S \odot}$ Jianan Zhang, ${ }^{\ddagger} \S$ Elena Alonso-Redondo, ${ }^{\dagger}$ Rebecca Sainidou, " Pascal Rembert," Krzysztof Matyjaszewski, ${ }^{\circledR \odot}$ Michael R. Bockstaller,*,*0 and George Fytas*,

${ }^{\dagger}$ Max Planck Institute for Polymer Research, Ackermannweg 10, 55128 Mainz, Germany

${ }^{\ddagger}$ Department of Materials Science and Engineering, Carnegie Mellon University, 5000 Forbes Avenue, Pittsburgh, Pennsylvania 15213, United States

${ }^{\S}$ Chemistry Department, Carnegie Mellon University, 4400 Fifth Avenue, Pittsburgh, Pennsylvania 15213, United States

"Normandie Univ, UNIHAVRE, Laboratoire Ondes et Milieux Complexes, UMR CNRS 6294, University of Le Havre, 75 Rue Bellot, 76600 Le Havre, France

\section{Supporting Information}
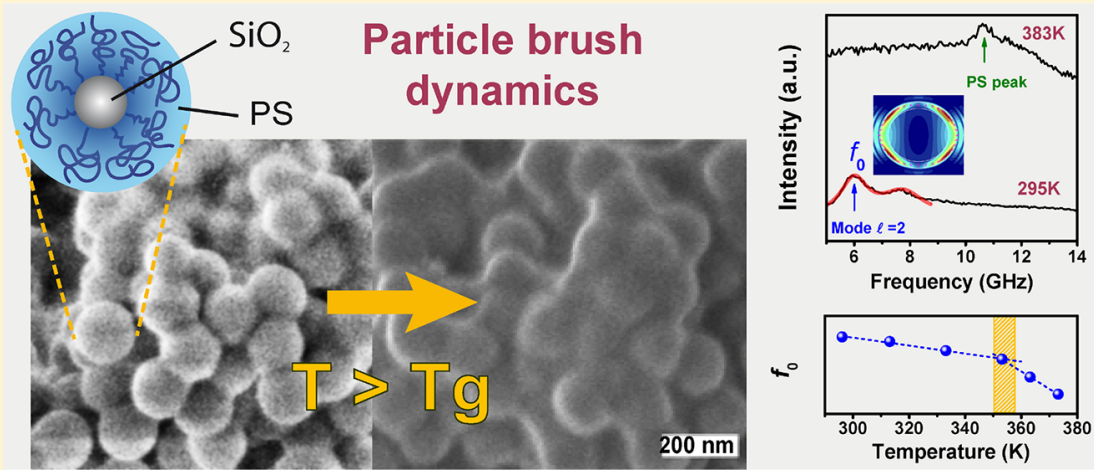

ABSTRACT: Polymer-tethered colloidal particles (aka "particle brush materials") have attracted interest as a platform for innovative material technologies and as a model system to elucidate glass formation in complex structured media. In this contribution, Brillouin light scattering is used to sequentially evaluate the role of brush architecture on the dynamical properties of brush particles in both the individual and assembled (film) state. In the former state, the analysis reveals that brush-brush interactions as well as global chain relaxation sensitively depend on grafting density; i.e., more polymer-like behavior is observed in sparse brush systems. This is interpreted to be a consequence of more extensive chain entanglement. In contrast, the local relaxation of films does not depend on grafting density. The results highlight that relaxation processes in particle brush-based materials span a wider range of time and length scales as compared to linear chain polymers. Differentiation between relaxation on local and global scale is necessary to reveal the influence of molecular structure and connectivity on the aging behavior of these complex systems.

\section{INTRODUCTION}

When polymers are densely tethered to the surface of particles to form so-called "particle brush" materials, then the mutual effect of surface-segment and excluded volume interactions alter the segment volume profile and can give rise to novel properties in materials that are assembled from brush particles. The resulting one-component hybrid materials have attracted interest as platform to fabricate hybrid materials with novel mechanical, dielectric, thermal, or phononic properties. ${ }^{1-8}$ The common thread in this emerging field of particle brush materials is that brush architectures are engineered such as to enable brush interdigitation and chain entanglement while maintaining well-defined microstructures in self-assembled films. The absence of particle aggregation in the resulting one-component hybrid materials also renders particle brush materials model systems to evaluate the role of chemical confinement on the aging behavior of polymer nanocomposite films. "Aging" refers to the gradual transition of amorphous polymer systems to more equilibrium-type structures by dynamical processes that involve segmental relaxation and the associated drift of physical properties. ${ }^{9}$ The pervasive relevance of aging on material performance along with its role in understanding structure and dynamical properties of condensed

Received: August 14, 2017

Revised: October 3, 2017

Published: October 30, 2017 
matter has rendered "aging" and "glass formation" one of the most actively researched areas in polymer physics. ${ }^{10-12}$

Previous work has shown that polymer relaxation in nanocomposite materials sensitively depends on chain length, the distance between dispersed particle fillers, and particlepolymer interactions. ${ }^{13}$ In binary particle-in-polymer dispersions, the latter two quantities are distributed and mutually dependent, and thus, the selective analysis of each governing parameter is difficult. ${ }^{14-16}$ In contrast, the microstructure of one-component hybrid materials is uniquely defined by the brush architecture, i.e., the degree of polymerization and grafting density of tethered chains as well as the particle size and shape. To understand the effect of brush architecture on the glass formation process, Vaia and co-workers reported a calorimetric study of the glass transition in sparse and dense particle brush films. ${ }^{17}$ The authors suggested glass formation in particle brush films to encompass two sequential processes, i.e., the initial freezing of cooperative relaxations that are determined by long-ranged polymer-particle interactions and the subsequent slowdown of local relaxations that depend on the segmental volume distribution within the brush particles. ${ }^{17}$ The latter is expected to depend on the geometric confinement (and hence grafting density) of tethered chains; however, only a weak dependence of glass formation on the graft architecture could be determined. This is a surprising result given that previous studies on thin film polymers reported a pronounced effect of confinement on the aging behavior of polymers. ${ }^{18,19}$ In part, this might be attributed to the limited capacity of calorimetric techniques to distinguish dynamic modes other than local segmental motion that depend on the glass-rubber relaxation process. This is particularly limiting in complex structured systems such as particle brush assemblies in which relaxations involve dynamic processes on both the local as well as the collective global level that span over multiple time and length scales. ${ }^{20}$

Brillouin light scattering (BLS) presents a powerful complementary technique to study the glass transition process in complex structured materials. The versatility of BLS to analyze glass formation originates from the sensitivity of thermal phonons to local packing and interactions that determine the thermoelastic properties of a medium at long wavelengths, i.e., in the linear acoustic regime. ${ }^{3,21}$ For (shorter) phonon wavelengths that are commensurate with the characteristic length scales of the system, the phonon dispersion (i.e., the dependence of mode frequency on the scattering wave vector) is a sensitive indicator of the flow of thermoelastic energy. ${ }^{3}$ Two experimental quantities are hence obtained that provide complementary information about the dynamical processes underlying the glass transition: the adiabatic longitudinal speed of sound (obtained through the low wave vector $\mathbf{q}$ limit) that senses the glass transition through compressibility and isobaric thermal expansion and the particle eigenmode frequencies (obtained by analysis of particle elastic vibrations). ${ }^{22-26}$ The lowest frequency, which depends on the transverse speed of sound and the particle's characteristic length scales, probes glass transition mainly via cohesive forces and dilatometry effects. By representation of the eigenmode frequencies with elastic phonon scattering theory, a detailed picture can be obtained about the spatial localization of the elastic energy that sheds light into the softening of the fundamental vibration modes at the glass transition in complex systems.

In this contribution we illustrate the application of BLS to evaluate the effect of graft architecture on the glass-rubber transition in particle brush assembly structures. We demonstrate that the analysis of the eigenmode characteristics of isolated particles as well as the effective medium longitudinal sound velocity in contiguous particle brush films gives information about the local segmental response in the glass transition as well as the impact of chain dynamics on the particle vibrations. Moreover, the concurrent analysis of these quantities reveals the sensitive influence of the grafting density on the dynamic response of the system near the glass-rubber transition. Specifically, the glass transition in sparsely tethered systems is found to cause a sharper and more pronounced change in the phonon lifetime. The latter is similar to pristine homopolymer systems and is shown to alter film formation kinetics. In contrast, in densely grafted systems a more gradual and suppressed transition is observed. These different signatures are interpreted as a consequence of the more pronounced stretched chain conformation in dense systems and their associated effect on segment volume profiles and relaxation.

\section{RESULTS AND DISCUSSION}

The material system in the present study consists of polystyrene (PS) grafted silica particles of radius $R_{\mathrm{c}}=57 \pm 3$ nm with systematically varying architecture (grafting density $\sigma$ and degree of polymerization of tethered chains, $N$ ) that were synthesized via surface-initiated atom transfer radical polymerization (SI-ATRP) using established protocols. To evaluate the role of segmental distributions, the grafting density of particle brushes was varied from 0.08 to $0.6 \mathrm{~nm}^{-2}$. Four particle brush systems are included in the present study that will subsequently be referred to as "sparse" and "dense" brush particles. Here we note that the classification of "sparse" or "dense" is based not only on the nominal value of the grafting density but also on the dependence of brush height on the degree of polymerization of tethered chains (see Figure 1). The characteristics are for sparse: $N=530, \sigma=0.27 \mathrm{~nm}^{-2}$ (sample ID: DP530) and $N$ $=1170, \sigma=0.08 \mathrm{~nm}^{-2}$ (sample ID: DP1170); and for dense: $N$ $=1300, \sigma=0.53 \mathrm{~nm}^{-2}$ (sample ID: DP1300) and $N=2480, \sigma$ $=0.39 \mathrm{~nm}^{-2}$ (sample ID: DP2480). A summary of all particle brush sample characteristics is presented in Table 1 .

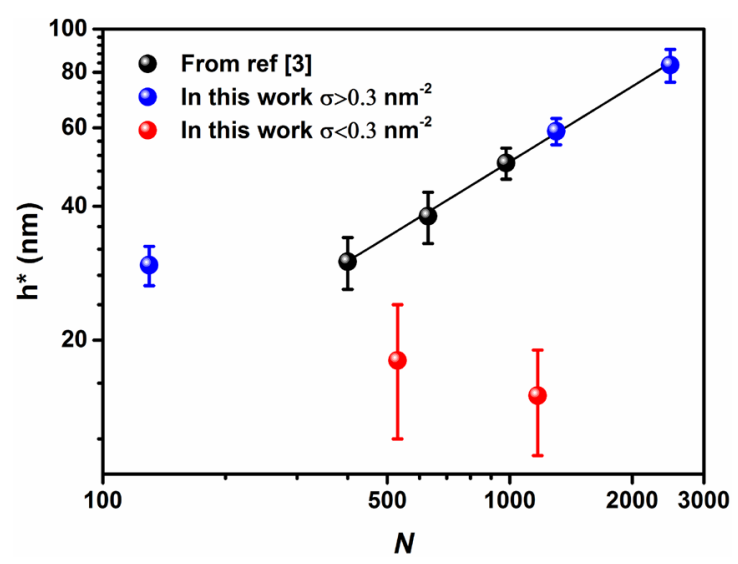

Figure 1. Thickness of polystyrene (PS) brush layer of PS-tethered silica particles obtained from their SEM images (Figure S1) for sparsely (red symbols) and densely (blue symbols) grafted polymer chains. Black symbols denote values reported previously for similar brush particle systems that were obtained for the identical silica particle batch and high grafting density (adopted from ref 3 ). 
Table 1. Molecular Parameters of the Polystyrene-Tethered Silica Particles ${ }^{a}$

\begin{tabular}{|c|c|c|c|c|c|}
\hline \multirow[b]{2}{*}{ sample } & \multicolumn{2}{|c|}{$\begin{array}{c}\text { grafting } \\
\text { characteristics }\end{array}$} & \multirow{2}{*}{$\frac{\text { particles }}{h^{*}(\mathrm{~nm})}$} & \multirow{2}{*}{$\frac{\text { films }}{h^{\#}(\mathrm{~nm})}$} & \multirow[b]{2}{*}{$\phi_{\mathrm{PS}}$} \\
\hline & $\sigma\left(\mathrm{nm}^{-2}\right)$ & $N$ & & & \\
\hline DP1300 & 0.53 & 1300 & $59 \pm 4$ & $63 \pm 8$ & $0.86 \pm 0.04$ \\
\hline DP2480 & 0.39 & 2480 & $83 \pm 7$ & $87 \pm 12$ & $0.93 \pm 0.03$ \\
\hline DP530 & 0.27 & 530 & $18 \pm 6$ & $19 \pm 3$ & $0.56 \pm 0.02$ \\
\hline DP1170 & 0.08 & 1170 & $15 \pm 4$ & $11 \pm 6$ & $0.50 \pm 0.02$ \\
\hline
\end{tabular}

${ }^{a} h^{*}$ : brush height determined by SEM images in Figure S1; $h^{\#}:$ brush height determined by TEM images in Figure $S 6$; $\phi_{\mathrm{PS}}$ : volume fraction of PS is determined by different methods (see Table S1).

Tethered Chain Dimensions. To evaluate the conformational state of surface-tethered chains, the brush height of pristine isolated particle brushes $h^{*}$ was determined by evaluation of particle dimensions using scanning electron imaging (Figure S1) and reported in Table 1. Figure 1 depicts $h^{*}$ as a function of $N$ for the particle brushes with the same silica cores studied in the present work and previous work. ${ }^{3}$ The data reveal that the PS thickness for polymer brush particles in air conforms to a scaling behavior $h^{*} \sim N^{0.56 \pm 0.03}$ for grafting densities greater than about $0.3 \mathrm{~nm}^{-2}$. Figure 1 also reveals that the sparsely grafted systems DP530 ( $\sigma=0.27$ $\left.\mathrm{nm}^{-2}\right)$ and DP1170 $\left(\sigma=0.08 \mathrm{~nm}^{-2}\right)$ display a significant reduction of brush height as compared to the densely tethered counterparts. The small brush height suggests a pronounced collapse of chain conformations. The chain collapse in the case of DP530 (and DP1170) motivates its classification as a "sparse brush" for the purpose of the present study. Note that both structural parameters $N$ and $\sigma$ differ for sparse brush particle systems, and thus a detailed scaling relation cannot be established for the sparse grafting density regime. The system DP100 $\left(\sigma=0.61 \mathrm{~nm}^{-2}, N=130\right)$ assumes a special role since its chains are more stretched than other dense brush systems based on the scaling of Figure 1 (solid line). In fact, light scattering studies reveal that the brush dimension approaches its size in dilute solution (not shown here). It is interesting to note that the scaling characteristics differ in dense tethered systems (DP100, DP1300, and DP2480) dependent on the degree of polymerization of tethered chains. The trend is consistent with previous reports and can be attributed to the more pronounced packing constraints that act on polymer segments in the vicinity of the particle surface. ${ }^{2}$

The distinct conformation of sparsely grafted chains should impact the dynamics of the individual particles and the thermomechanical properties of their assembled films. Figure 1 illustrates an important inherent feature of brush particles; that is, the brush height (and hence chain conformations) is a function not only of $N$ but also of grafting density, $\sigma$. These quantities are thus expected to govern the interactions and dynamical features of brush particles in pristine (individual) as well as assembled (film) form. We address first the effect of brush density on the eigenvibration spectrum as a function of temperature to illustrate the effect of packing on both chain and segmental dynamics. Subsequently, we discuss the role of graft density on film formation and relaxation.

Particle Vibration Spectra. BLS is a powerful technique to record the thermal shape fluctuations of submicrometer size particles depending on their geometry, morphology, and mechanical properties. ${ }^{22,23}$ For nanoparticles with size less than $100 \mathrm{~nm}$ Raman scattering ${ }^{2 f}$ and pump-probe ${ }^{24}$ techniques can be utilized, but fewer modes are resolved as compared to BLS, rendering the analysis less unique. The vibration spectra of the densely PS-tethered silica particles $(\sigma \approx$ $0.6 \mathrm{~nm}^{-2}$ ) have been recently reported (black symbols in Figure 1). ${ }^{26}$ The eigenfrequencies revealed a red-shift compared to uniform core-shell particles that was interpreted as a consequence of the anisotropic elastic impedance mismatch across the polymer-particle interface. The origin of the mechanical anisotropy was argued to be the stretching of the tethered chains near the interface which is more pronounced for short and densely tethered chains. For similar grafting density, the effect was diluted with increasing degree of polymerization and hence PS volume fraction $\phi_{\mathrm{PS}}$.

Figure 2 displays vibration spectra at $295 \mathrm{~K}$ for grafted particles of Table 1 . The two dense particle brushes with $N=$
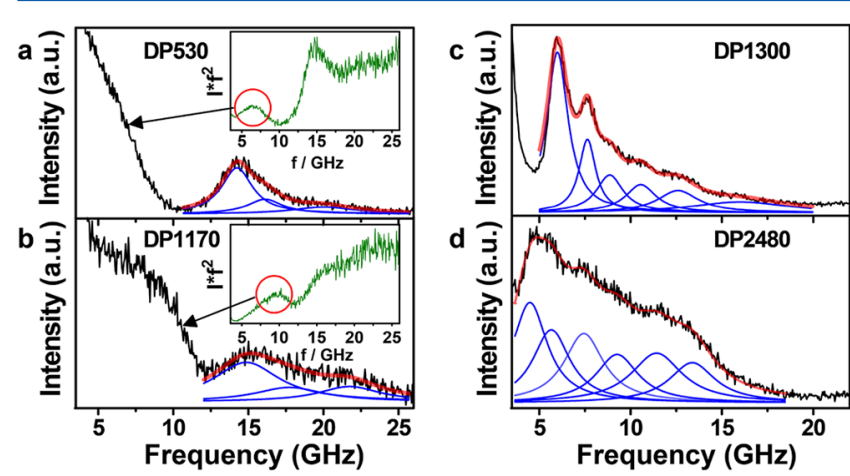

Figure 2. Eigenmode spectra of DP530 (a), DP1170 (b), DP1300 (c), and DP2480 (d) particles in a powder form at $295 \mathrm{~K}$ represented by several Lorentzians (blue solid lines). Power spectra for the DP530 and DP1170 are shown as insets in (a) and (b), and the mode $(l=1)$ is indicated by arrows in the main panels a and $b$. This mode is absent in DP1300 and DP2480.

1300 and 2480 (c, d) display a rich spectrum mainly due to their larger diameter, whereas for the other two sparsely grafted systems ( $a, b)$ the lower magnification, $q d \sim O(1)$, implies that only low-frequency modes can be detected. ${ }^{22}$ The spectra in Figure 2a,b display a low-frequency shoulder due to adhesion of neighboring particles (Figure S1) that transforms the translational dipole $(l=1)$ mode, also known as rigid-body-resonance (RBR), within isolated particles into a vibration-like mode that is characteristic for particle twins. ${ }^{25,28,29}$ This mode is clearly seen in the power spectra: intensity times frequency squared (insets to Figure $2 \mathrm{a}, \mathrm{b})$. Its frequency, $f_{11} \sim(k / \mathrm{m})^{1 / 2}$ with $k$ and $m$ being the effective spring constant and particle mass, respectively, is $7 \mathrm{GHz}$ for DP530 and $10 \mathrm{GHz}$ for DP1170. The ratio between these frequencies slightly exceeds the expected $m^{-1 / 2}$ dependence assuming the tethered PS mass (see Figure $3 \mathrm{~b}-\mathrm{e})$ and suggests somewhat stronger $k$ for DP1170. The absence of this low-frequency mode $(l=1)$ in the vibration spectra of DP2480 and DP1300 particles (Figure 2c,d) is attributed to the red-shift of $f_{11}$ (higher $m$ and/or weak $k$ ). In fact, SEM images (Figure S1) suggest the particles rich in PS (DP1300 and DP2480) to exhibit a reduced degree of clustering compared to DP530 and DP1170 systems with lower PS weight fraction. A reliable resolution of $f_{11}$ requires sufficiently strong particle adhesion $(\sim k)$ so that this mode can be separated from the strong elastic Rayleigh peak. ${ }^{22,28,29}$ The reduced frequency of cluster formation in the case of dense brush systems suggests that $k$ is larger for sparse systems. Indeed, this is consistent with prior reports on the assembly 


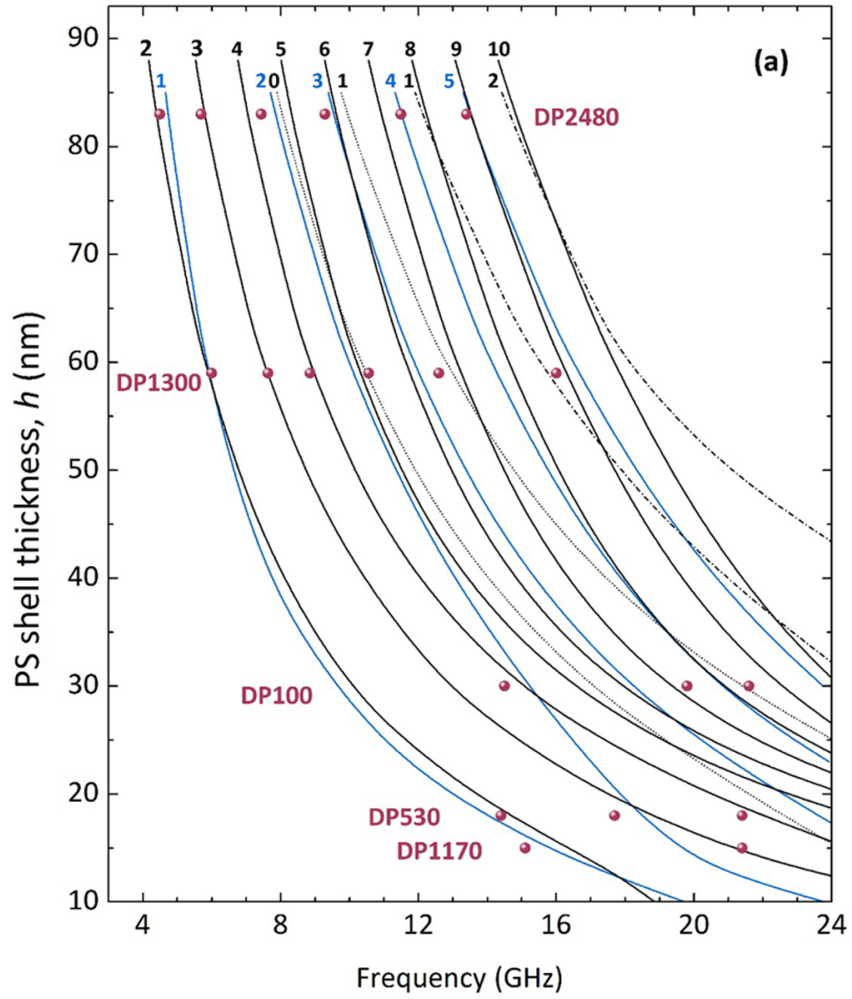

(b)

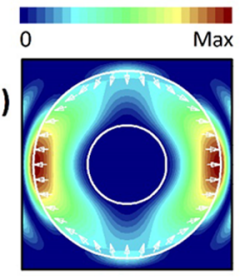

(c)

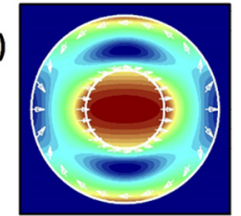

(d)

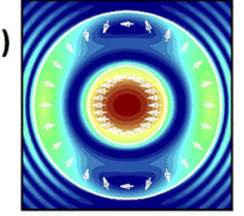

(e)

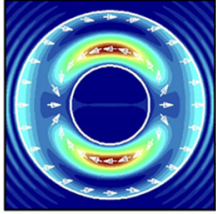

Figure 3. (a) Calculated dispersion relation of the spheroidal eigenmodes for $\mathrm{SiO}_{2}-\mathrm{PS}$ particles immersed in air, consisting of a silica core of radius $R_{c}=57 \mathrm{~nm}$ and a polystyrene shell of thickness $h$. The symbols denote the experimentally resolved eigenfrequency positions for the five samples. The theoretical calculations predict four spheroidal subfamilies: shell-localized mostly radial vibrations (solid black lines), mostly tangential, and mostly radial vibrations localized in both the shell and core (blue solid and dotted lines, respectively) and shell-localized mostly tangential vibrations (dash-dotted lines). The angular momentum quantum number $l$ characterizing each of these modes is given at the top of the plot. Right panel: representative displacement field plots of the first mode $f_{n, l}$ of these families for $h=83 \mathrm{~nm}: f_{n=1, l=2}=4.39 \mathrm{GHz}(\mathrm{b}), f_{n=2, l=1}=4.73 \mathrm{GHz}(\mathrm{c}), f_{n=3, l=1}=$ $9.94 \mathrm{GHz}(\mathrm{d})$, and $f_{n=4, l=1}=12.12 \mathrm{GHz}(\mathrm{e})$. The color maps correspond to the square modulus of the scattered displacement field at a cut passing at the center of the sphere (incidence is considered along the horizontal axis from left to right), and the arrows show the direction of the displacement field at selected positions.

behavior of sparse brush systems that revealed the formation of complex particle brush aggregate structures in film and composite systems. ${ }^{15,16,30}$

Theoretical calculations using multipole expansion of the elastic field for an individual core-shell $\mathrm{SiO}_{2}-\mathrm{PS}$ particle immersed in air, with ordinary (perfect) boundary conditions (PBC) applied at the silica-PS interface, ${ }^{31,32}$ ascribe the experimentally observed eigenmodes to the spheroidal vibrations of the particle. The spherical symmetry of the latter implies that the elastic field is expanded into a vector spherical wave basis that can be classified into two uncoupled subspaces: spheroidal and torsional solutions. The corresponding eigenfrequencies, $f_{n}$, are characterized by the angular momentum quantum number $l$ and their order of appearance $n$ with increasing frequency.

In Figure 3 we show the calculated dispersion relation of the spheroidal vibrations of the particle as a function of the thickness $h$ of the PS shell coating assuming a fixed silica core of radius $R_{\mathrm{c}}=57 \mathrm{~nm}$; both materials are assumed to be homogeneous and isotropic. Bulk elastic parameters are used for PS (mass density $\rho=1050 \mathrm{~kg} / \mathrm{m}^{3}$, longitudinal and transverse elastic velocities $c_{\mathrm{L}}=2350$ and $c_{\mathrm{T}}=1210 \mathrm{~m} / \mathrm{s}$, respectively); for silica we employ the same elastic parameters as they were determined in previous studies. ${ }^{26}$ The calculated spheroidal resonance frequencies are red-shifted with increasing PS shell thickness $h$ (Figure 3a), and the corresponding vibrational modes are classified into four different subfamilies with respect to their spatial localization inside the particle (core-localized, shell-localized, or both) and/or their highamplitude elastic field direction (mostly tangential or mostly radial vibrations), as detailed by the different plot lines in Figure $3 \mathrm{a}$ with the corresponding field plots on resonance depicted in Figures $3 b-e$. We note in passing that when two dispersion lines of the same $l$ come close enough to each other, a hybridization occurs; i.e., each line loses its pure spatial localization character, and a mixing of these two vibrational states appears. This is the case in Figure $3 \mathrm{a}$ for the two $l=2$ dispersion curves (black and blue solid lines) coming closer and interacting at about $h \sim 40 \mathrm{~nm}$.

Comparison with the experimental frequency values (symbols in Figure 3a) for the different samples and for $h$ taken from Table 1 shows a good agreement and assigns the first few strong low-frequency modes in DP2480 and DP1300 to PS-localized and mostly radial, spheroidal modes with $l=2-$ 5. For the two sparsely tethered particle systems, the two resolved modes are assigned to $l=2-4$ for DP530 and $l=2,3$ for DP1170 of the same subfamily of spheroidal modes. Spheroidal modes belonging to the other three subfamilies (PSlocalized tangential, core-localized, and coupled-core-shell tangential or radial vibrations) do not seem to strongly contribute to the BLS recorded spectrum. A clear physical picture explaining the preference of certain modes to be BLS active requires a more thorough theoretical analysis in the future by evaluating the relation between the BLS intensity and 
the symmetry and spatial distribution of the elastic-field virtual bound states inside particles. An exception to the good agreement between experimental and calculated particle eigenmodes seen in Figure 3a is the case of the DP100 system in its powder state. Here, the first experimentally observed resonance peak at about $14.2 \mathrm{GHz}$ is found in the vicinity of the theoretical $l=4$ mode. The origin for the stark difference in the resonance behavior of the DP100 system is currently unknown. One could argue that the $l=2$ mode is not BLS active, for example, because of possible anisotropic elasto-elastic and/or elasto-optic behavior. ${ }^{33}$

We note that other types of boundary conditions (such as imperfect boundary conditions) that were introduced previously cannot account for the observed difference in resonance behavior. ${ }^{26}$ It should be mentioned that the lowest spheroidal resonance frequency $f_{l=2}$ mode for the core-shell particle under study can be accurately estimated by the formula $f_{l=2}=$ $0.405 c_{\mathrm{T}}^{0.84} h^{-0.81}$ for shell thickness $h$ varying from 30 to $80 \mathrm{~nm}$ and for PS velocity ratios (with respect to the corresponding bulk values) ranging from 1.00 to 1.10 . For the sake of completeness, we note that in the case of homogeneous spherical particles of radius $R f_{l=2}$ was found to follow the Lamb expression $f_{l=2}=0.425 c_{\mathrm{T}} R^{-1}$.22,34

The successful representation of the particle vibration spectra in the glassy state with PBC using bulk PS elastic constants indicates a core/shell behavior for all four high $N$ particle brush systems. Based on Figure 1, the low-grafting-density systems (DP530 and DP1170) exhibit less extended chain conformations as compared to the high-density counterparts (DP1300 and DP2480). This is revealed by the presence of interactioninduced low frequency $(l=1)$ mode (Figure $2 a, b)$ that suggests entanglement between tethered chains and confirms previous reports that have demonstrated high grafting densities to hinder interpenetration of stretched tethered chains. ${ }^{35-37}$ Analysis of the temperature dependence of vibration spectra sheds light into the nature of these interactions and allows the estimation of the glass transition temperature, $T_{\mathrm{g}}$, of the tethered PS chains.

Figure 4a,c displays the eigenmode spectra of DP530 and DP1300 at different temperatures in the range 295-425 K exceeding the $T_{\mathrm{g}}(\sim 370 \mathrm{~K})$ of the bulk PS (for the other two high $N$ systems, see Figure S2). The eigenmode spectrum persists as long as individual particles resist the interdiffusion that eventually leads to film formation and disappearance of the eigenmodes $^{28}$ of the individual isolated particles in air. ${ }^{26}$ The information contained in the BLS spectra can be used to extract indirectly the glass transition temperature which is defined in these cases as the temperature at which the slope of the sound velocities $\left(c_{\mathrm{L}}\right.$ and $c_{\mathrm{T}}$ ) changes (i.e., $\mathrm{d} c_{\mathrm{L} / \mathrm{T}} / \mathrm{d} T$ is discontinuous). ${ }^{38}$ In the eigenmode spectra shown in Figure $4 \mathrm{a}, \mathrm{c}$, the first few strongest eigenmodes can be easily resolved by Lorentzian-shaped fitting curves; each mode is characterized by its frequency position $f_{\mathrm{R}}$ and line width $\Gamma$. The variation with temperature of these two quantities, $f_{\mathrm{R}}(T)$ and $\Gamma(T)$, depicted in Figures $4 \mathrm{~b}$ and $4 \mathrm{~d}$, is of importance to deduce the glass transition. ${ }^{38-41}$

Before analyzing in details the $T$-dependent characteristics of the BLS spectra of our samples, two aspects should be considered: First, both elastic velocities $c_{\mathrm{L}}=(M / \rho)^{1 / 2}$ and $c_{\mathrm{T}}=$ $(G / \rho)^{1 / 2}$ depend on the mass density $(\rho)$ and longitudinal $(M)$ and shear $(G)$ moduli. The very similar temperature dependence of $c_{\mathrm{L}}(T)$ and $c_{\mathrm{T}}(T)$ in the glassy state leads to a robust $T_{\mathrm{g}}$ value (Figure S3), suggesting that the $T$ dependence of the
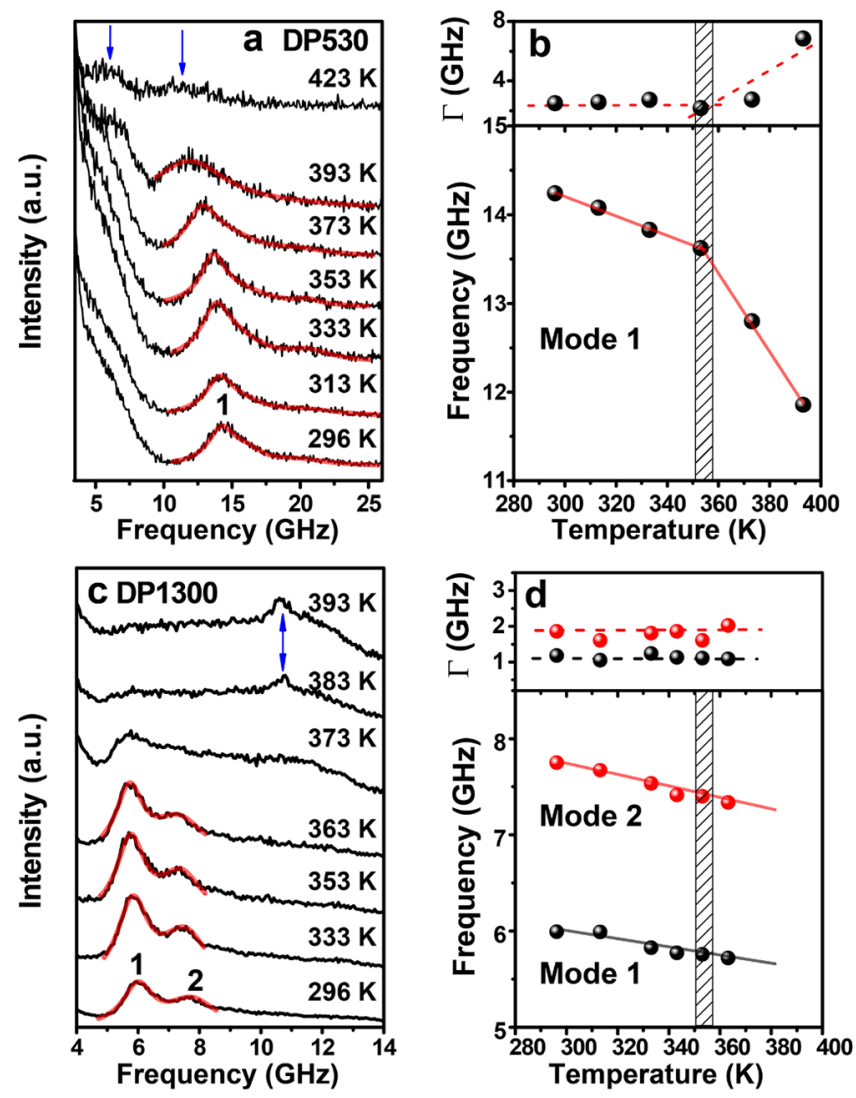

Figure 4. Eigenmode spectra of DP530 (a) and DP1300 (c) measured at different temperatures in the glassy and rubbery PS state. The peak frequency (lower panel) and line width (upper panel) of the strongest modes are determined by curve fitting with Lorentzian functions (red curves) and depicted as a function of temperature for DP530 (b) and DP1300 (d). The vertical shaded area indicates the glass transition region in DP530 (b), while for DP1300 (d) no transition was observed until $365 \mathrm{~K}$, above which no eigenmodes could be resolved. The acoustic phonon characterizing the formation of contiguous bulk-like films is indicated by blue arrows in the eigenmode spectra (at $q=$ $0.0118 \mathrm{~nm}^{-1}$ and backscattering in panels a and c).

cohesive forces is indeed the dominant factor. Because of the comparatively slow heating rate that is applied in the BLS measurements, $T_{\mathrm{g}}$ that is obtained from BLS is expected to be lower than the corresponding DSC value. Since the vibration frequency of the strongest $l=2$ mode is roughly proportional to $c_{\mathrm{T}} h^{-1}$ (see discussion of Figure 3) and given the weak influence of thermal expansion on the PS shell thickness $h$ $\left(\sim \rho^{-1 / 3}\right)\left(\alpha<10^{-4} \mathrm{~K}^{-1}\right),{ }^{42}$ the variation of frequency position in the $T$-dependent eigenmode spectra can be used to deduce the glass transition temperature $T_{\mathrm{g}}$ via mainly the $T$-dependent $c_{\mathrm{T}}$. Interestingly, the sound attenuation that is described by the mode line width $\Gamma(T)$ displays a nonmonotonic increase above $T_{\mathrm{g}}$ (Figure $4 \mathrm{~b}$ and Figure $\mathrm{S} 2 \mathrm{~b}$ ). This observation is rather unexpected because dissipation is negligible at gigahertz frequencies (since segmental relaxation times just above $T_{\mathrm{g}}$ are expected to be of the order of seconds). We relate this increased dissipation to the interaction-induced low frequency ( $l=1)$ RBR mode.

Analysis of $f_{\mathrm{R}}(T)$ and $\Gamma(T)$ above $T_{\mathrm{g}}$ reveals two distinct types of behavior of the particle systems (Figure 4 and Figure S2): type I (DP1300 and DP2480), characterized by the presence of a bulk PS peak and the smear-out of eigenmodes, and type II (DP530 and DP1170), characterized by the 
prevalence of eigenmodes above $T_{\mathrm{g}}$. Indeed, for the type I particle systems (high grafting density $\sigma$ and large PS volume fraction $\left.\phi_{\mathrm{PS}}\right)$ no eigenmode is discernible above $T_{\mathrm{g}} \approx 373 \mathrm{~K}$ (see Figures $4 \mathrm{c}$ and Figure S2c), which is confirmed by the appearance of the backscattering peak of the melt PS (the formation of a transparent film allows the observation of the acoustic phonon in PS at almost $180^{\circ}$ scattering angle). For DP1300 (Figure 4c) and DP2480 (Figure S2c), all modes but the strongest abruptly disappear at about $373 \mathrm{~K}$ (along with the concurrent appearance of the PS longitudinal phonon). The line widths are virtually $T$-independent (Figure $4 \mathrm{~d}$ and Figure S2d). In this case, $T_{\mathrm{g}}$ can be estimated by the appearance of the bulk PS peak (at about $11 \mathrm{GHz}$ ) between 363 and $373 \mathrm{~K}$. The appearance of the bulk peak is consistent with the formation of contiguous bulk-like film structures during heating of the material and indicates that significant chain mobility must exist over this $T$ range to facilitate film formation.

Next, for the particle systems of type II (low grafting density and near symmetric PS volume fraction), eigenmodes prevail above $T_{\mathrm{g}} \approx 355 \mathrm{~K}$. The glass transition is now estimated by the change in the rate of both frequency and line width of the main eigenmode with temperature. The presence of the lowfrequency $(l=1)$ peak is clearly seen in the power spectra $I(f) f^{2}$ of type II particle systems in Figure 5 and suggests adhesion between neighboring particles. ${ }^{28}$ The variation with temperature of the frequency of this interaction-induced $(l=1)$ RBR mode changes abruptly at a temperature being comparable
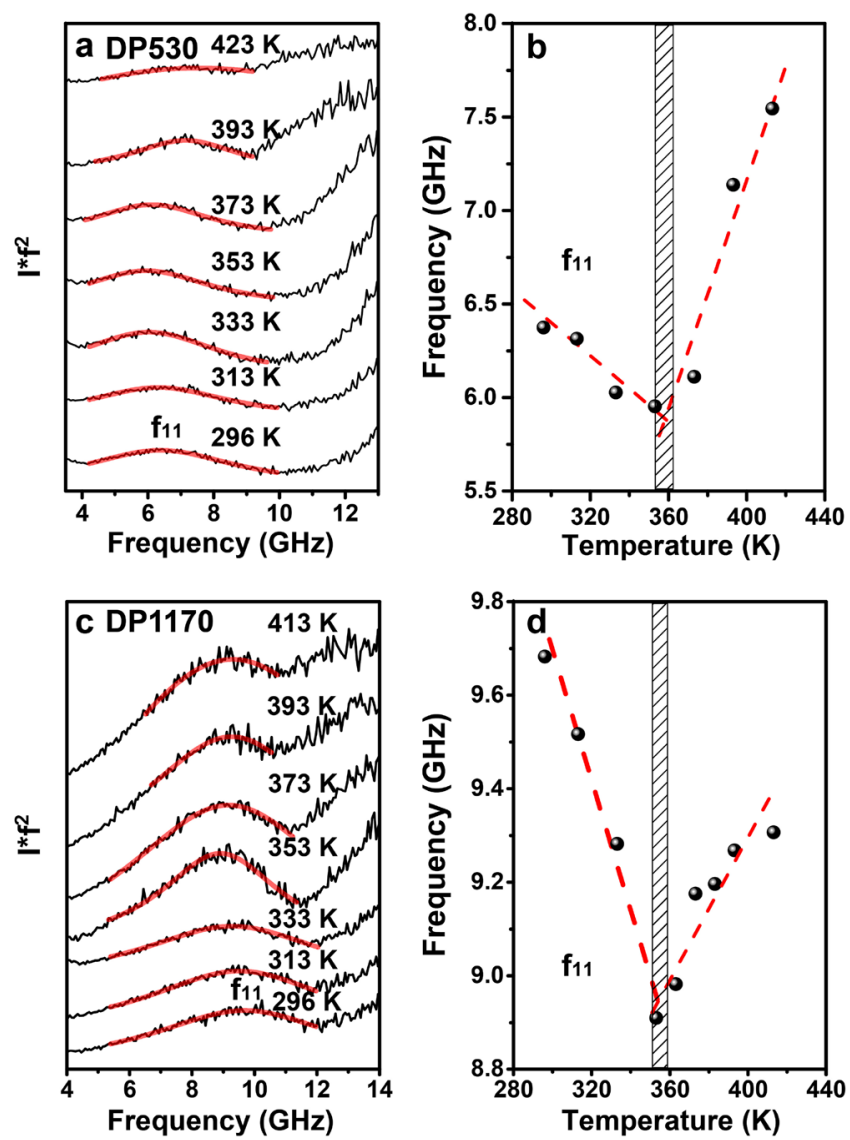

Figure 5. Power BLS spectra and the frequencies of the low frequency $(l=1)$ peak for $\operatorname{DP530}(\mathrm{a}, \mathrm{b})$ and $\operatorname{DP} 1170(\mathrm{c}, \mathrm{d})$ at different temperatures. The shaded area around $360 \mathrm{~K}$ indicates the glass transition region. to that of $f_{\mathrm{R}}(T)$ and $\Gamma(T)$ for the fundamental eigenmode. Moreover the slope $\left(\mathrm{d} f_{11} / \mathrm{d} T\right)$ changes from negative to positive at the transition temperature. All three experimental parameters indicate a lower $T_{\mathrm{g}}$ relatively to the bulk PS $\left(T_{\mathrm{g}} \sim\right.$ $373 \mathrm{~K}$ ) and the particles system of type I (Figure $4 \mathrm{~d}$ ). Few reports in the literature have investigated the glass transition behavior of bare nanoparticles in general ${ }^{43-45}$ and neat polymer-grafted nanoparticles. ${ }^{17,35,46}$ However, there is no solid conclusion on the effect of grafting density and polymer graft molecular weight on the average $T_{\mathrm{g}}$ response. In the most recent paper, a small $(1-2 \mathrm{~K}) T_{\mathrm{g}}$ increase compared to the free PS chains with the same molecular weight observed by DSC, whereas a $T_{\mathrm{g}}$ gradient from near the free surface to near the core interface was inferred by the fluorescence of molecular dye labeled at specific regions of the PS grafts. ${ }^{46}$

The dipole (RBR) mode is a sensitive indicator of interparticle adhesion. Since the "fusion" of polymer shells that is observed in the electron micrographs of "particle pairs" requires a sufficient fraction of relaxed chain segments (since only the latter are capable of entanglement and interpenetration), the RBR mode is sensitive to the graft density. The comparison of DP1170 to DP1300 (similar N) suggests that the reduced grafting density promotes chain entanglements and thus interparticle-segmental interactions. In type II systems (DP530 in Figure 4a and DP1170 in Figure S2a), the reduced chain mobility (caused by significant chain entanglements) renders the eigenmodes more robust. This is evidenced by the persistence of eigenmodes above $T_{\mathrm{g}}$ as revealed by the different slopes of $f$ vs $T$ but also $\Gamma(T)$. This trend is consistent with previous reports of a slowdown of the chain dynamics in the case of high grafting density systems due to arm retraction even for nonentangled chains (i.e., $N<N_{\mathcal{c}}$ where $N_{c}$ is the entanglement degree of polymerization). ${ }^{47}$ The different PS graft interpenetration is schematically shown in Figure 6.

For DP100 with predominantly stretched configuration no adhesion can be discerned as indicated by the absence of the RBR mode (Figure S4). This is consistent with the expectation that more pronounced stretching of tethered chains should

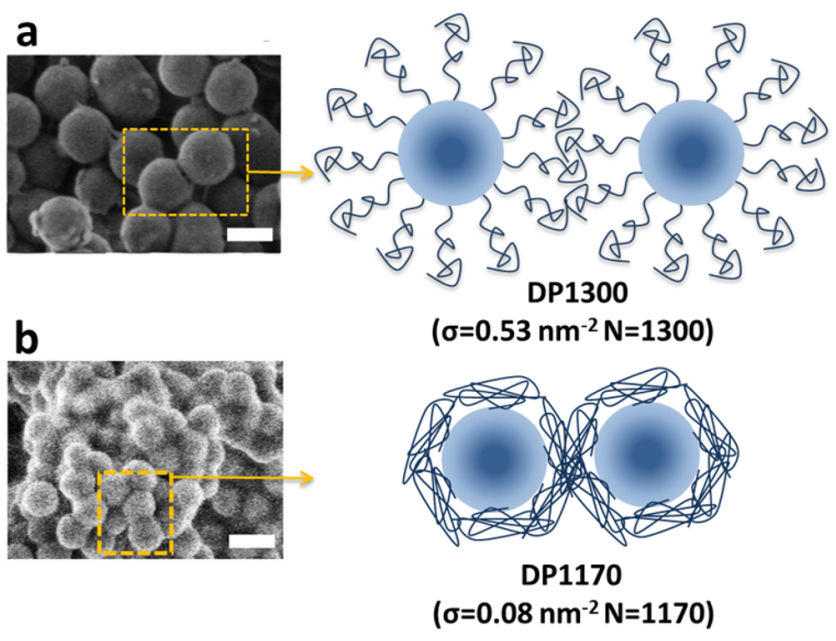

Figure 6. Scanning electron microscope (SEM) images (scale bar: 200 $\mathrm{nm}$ ) of two polystyrene (PS) grafted silica particles with similar degree of polymerization for the PS grafts but very different grafting density. The schematic presentation of the PS stretching and interpenetration of a pair of DP1300 (panel a, high grafting density and large interparticle distance) and DP1170 (panel b, low grafting density and short interparticle distance) is shown next to the SEM images. 

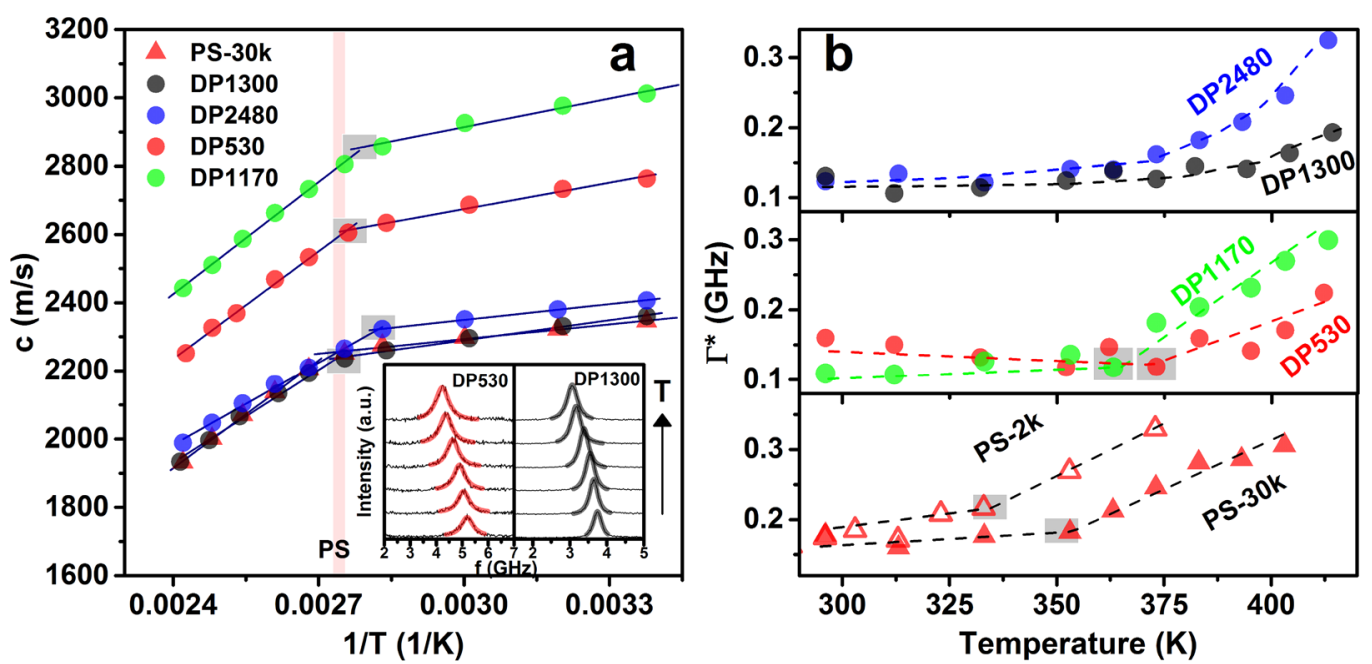

Figure 7. Temperature dependence of the longitudinal sound velocity (a) and line width $\Gamma^{*}(T)$ (b) for the four assembled DP films and bulk PS (values for $\Gamma^{*}(T)$ represent net values after subtraction of the instrumental contribution). Shaded areas indicate the estimated $T_{\mathrm{g}}$ from the two BLS experimental quantities. Two PS films with molecular weight $30 \mathrm{~K}\left(T_{\mathrm{g}} \approx 365 \mathrm{~K}\right.$ in panel a) and $2 \mathrm{~K}\left(T_{\mathrm{g}} \approx 337 \mathrm{~K}\right.$ in panel b) are displayed. Inset: typical BLS spectra for DP530 and DP1300.

impede brush interpenetration and hence retard film formation as indicated by the robust vibration modes in DP100 above $T_{\mathrm{g}}$. The chain stretching alters the local dynamics of DP100 as suggested by its higher $T_{\mathrm{g}}(373 \pm 2 \mathrm{~K}$, Figure S4) compared to the low-grafting density DP1170 and DP530 systems ( 354 K). Previous research on flat brushes (high grafting density) reported an increase of $T_{\mathrm{g}}$ that was attributed to the slowdown of the chain dynamics due to the reduced conformational entropy of the stretched chains. ${ }^{48,49}$ The chains progressively adopt Gaussian conformation with decreasing grafting density leading to an increased contribution of the entropy of the more mobile chains and hence to a $T_{\mathrm{g}}$ reduction relative to the bulk state. ${ }^{48}$ Furthermore, a heuristic similarity of the stretched DP100 and collapsed individual type II particle brushes (DP530 and DP1170) to free-standing and supported films, respectively, might find support from the reduced $T_{\mathrm{g}}$ in the type II systems.

The preceding information on the thermomechanical behavior of the individual polymer-tethered particles is based on the volume thermal expansion and not directly on the polymer segmental dynamics. The latter is frozen at $T_{\mathrm{g}}$ and is at the origin of the specific heat relaxation probed by DSC, which has revealed pronounced confinement effects (i.e., a reduction of the glass temperature) in submicrometer PS spherical colloids. ${ }^{43,50}$ In the few reports on particle brush systems ${ }^{5,35}$ and PS brushes, ${ }^{11,46,51} T_{\mathrm{g}}$ was found to be virtually independent of the brush thickness. To gain insight into the origin of the apparent reduction of the glass transition temperature in the case of brush particles, the $T_{\mathrm{g}}$ of bulk particle brush materials was determined by DSC. To facilitate conditions close to BLS experiments, particle brush solutions were precipitated in nonsolvent (ethanol) and subsequently dried in a vacuum. Three consecutive DSC runs $(298 \mathrm{~K}<T<403 \mathrm{~K}$ at a heating (cooling) rate $1 \mathrm{~K} / \mathrm{min}(10 \mathrm{~K} / \mathrm{min})$ and $10 \mathrm{~min}$ equilibration at maximum temperature) were performed for each sample. The first heating run corresponds to individual particles in the powder resembling closely the BLS experiment, whereas a progressive transformation to contiguous films was observed during the two subsequent runs. The glass transition was determined from heating curves for consistency with BLS experiments.

As revealed by the data shown in Table S2, all particle brush samples (with the exception of DP1300) show a distinctive increase of $T_{\mathrm{g}}$ with increasing number of heating/cooling cycles. In as-prepared particle brush powder samples several transitions are observed, the most distinctive transition being a glass transition at about $353 \mathrm{~K}$ (close to the transition observed in BLS). With increasing number of heating/cooling cycles the number of detectable transitions decreases (see also the DSC traces shown in Figure S5). In the case of DP1170 and DP2480 only one major transition at $T \sim 382 \mathrm{~K}$, close to the expected glass transition temperature of PS, is observed after the first cycle. DP530 exhibits a similar behavior albeit the signature of the lower temperature transition is retained even after three runs. The $T_{\mathrm{g}}$ of the DP1300 system shows only a modest increase to $T_{\mathrm{g}} \sim 355 \mathrm{~K}$; however, it should be noted that a more pronounced increase (to $378 \mathrm{~K}$ ) was observed after (vacuum) annealing of the sample at $T \sim 398 \mathrm{~K}$ for 3 days, during which the brush particles coalesced to form a contiguous film. The DSC traces shown in Figure S5 reveal the implications of the gradual transition from the individual brush particle to the contiguous film state on the thermal behavior of the system. Multiple thermal transitions in the individual brush particle state (first run) converge to a single transition at about $382 \mathrm{~K}$ after repeated cycling through the glass transition (third run). The glass transition temperature measured after three runs was indistinguishable from the $T_{\mathrm{g}}$ of films after 3 days of thermal annealing.

Comparison of the DSC (Table S2) and BLS (Table S3) results suggests that the comparatively lower transition temperatures that are observed in BLS originate from the pronounced far-from-equilibrium local structure of powder particle brush systems in the as-prepared state. In particular, the high surface energy of air/polymer interfaces causes the collapse of tethered chains, as can be verified by the reduced scaling of brush height and degree of polymerization (Table 1). Evidently, this collapsed state results in a lowering of $T_{\mathrm{g}}$. To realize more ideal chain conformation in the film or melt state, dynamic processes must occur on the chain and particle level. 
The slow particle dynamics hinders transitions during the annealing process and delays the "convergence" of $T_{\mathrm{g}}$ during DSC as compared to linear chain analogues.

Assembled Particle Films. To gain a better understanding of the role of brush architecture and chain conformation on the relaxation behavior of contiguous films, BLS was performed on equilibrated film samples. The conformation of the tethered PS chains depends on the particle environment, i.e., either air (for individual brush particles) or the polymer-filled interstitial space between particles (in the case of contiguous assembled films). A direct consequence of film formation is the disappearance of the eigenmodes in the BLS spectrum and the emergence of rich phononic dispersion. ${ }^{3}$ Here, the glass dynamics can be inferred by examination of the temperature dependence of the effective medium (long wavelength) sound velocity. To perform this analysis, we assume that contiguous particle brush films can be considered as ideal nanocomposites, free of phase separation and metastability. ${ }^{14,52}$ Using BLS, the $T_{\mathrm{g}}$ of bulk polymers ${ }^{38}$ can be determined from the onset temperature at which the slope of $c(T)$ decreases or increases (depending on whether cooling or heating runs are performed). Here we note that the rates for the two speeds of sound (longitudinal and transverse) are virtually the same below and different above $T_{\mathrm{g}}$ as can be deduced by the change of the Poisson's ratio at $T_{\mathrm{g}}$ in the case of a PS film (Figure S3). The temperature dependence of the longitudinal sound velocity, $c_{\mathrm{L}}$, for the four assembled particle films (Figure S6) and a PS film (molecular weight $30 \mathrm{~K}$ ) that is used as a reference is shown in Figure 7 . We should note that these traces represent the second heating cycle, i.e., after heating the films to $400 \mathrm{~K}$, equilibration for $30 \mathrm{~min}$, and subsequent slow cooling at room temperature overnight; all first heat runs revealed low $T_{\mathrm{g}}$ 's that could not be unequivocally identified. The particle brush films do not change morphology after heating above $T_{\mathrm{g}}$. To confirm this assertion, the microstructure of particle brush films was assessed by two methods: First, the comparison of BLS spectra of films measured at $295 \mathrm{~K}$ before and after glass transition experiments. The similarity of spectra provides strong indirect evidence for the similarity of the microstructure. Second, the evolution of microstructure of (monolayer) particle brush films during thermal annealing using TEM did not show an indication of structural changes on time scales relevant to the experiments in our study (see Figure S7).

Figure 7 reveals that bulk PS and nanocomposite films display distinctive changes in both longitudinal sound velocity and phonon lifetime $\left(1 / \Gamma, \Gamma=q^{2}(\eta / \rho)\right)$ at $T_{\mathrm{g}}$. The rate of the change of longitudinal sound velocity with temperature, $\mathrm{d} c_{\mathrm{L}} / \mathrm{d} T$ $=\left(\partial c_{\mathrm{L}} / \partial V\right)_{T}(\partial V / \partial T)_{P}$, is mainly determined by cohesive forces and dilatometry effects, whereas that of the phonon lifetime, $\partial \Gamma(T) / \partial T) \sim \partial \eta(T) / \partial T$, relates to the local dynamic viscosity $(\eta / \rho)$ (at hypersonic frequencies). ${ }^{53}$ Interestingly, $T_{\mathrm{g}}$ obtained from $c_{\mathrm{L}}(T)$ assumes quite similar values (close to bulk PS film) for all nanocomposite films (Figure 7a and Table S3). As all the films are free-standing with thickness in the micrometer scale, negligible confinement of the PS chains is anticipated in these systems. ${ }^{18}$ Furthermore, all particle brush systems consist of the same submicrometer size silica cores, and hence all these single component composites exhibit similar $T_{\mathrm{g}}$ values. We speculate that the effect of chain stretching on $T_{\mathrm{g}}$ might be weakened due to the different environment (air in the case of the individual particles vs PS theta matrix in the assembled films). The difference in $T_{\mathrm{g}}$ among the assembled particle brush films becomes small, whereas the phonon lifetime shows a sensitive temperature dependence that is a function of grafting density. Type I systems (DP1300 and DP2480) display a monotonic increase with temperature while for type II systems (DP530 and DP1170) a distinctive change of $\Gamma^{*}(T)$ is observed at $T_{g}$, similar to bulk PS. To interpret the above observation, we recall that phonon attenuation (which is proportional to $\Gamma^{*}(T) / q^{2}$ ) relates to dissipation either due to dynamic viscosity or phonon scattering. The latter should be rather insensitive to the glassrubber transition, as no structural changes are anticipated, and hence the local viscosity $(\eta / \rho)$ does appear responsible for the nonmonotonic change of $\Gamma^{*}(T)$ around $T_{\mathrm{g}}$. In this case, a nonmonotonic change of $\Gamma^{*}(T)$ is expected at $T_{\mathrm{g}}$. This is indeed observed for bulk PS and type II systems. The analogue trend in $\Gamma^{*}(T)$ for sparse brush particles and the reference bulk PS thus further confirms similar chain conformations. The behavior is different for type I brush particles (DP1300 and DP2480) which do not show a distinctive change of $\Gamma^{*}(T)$ near the glass transition. We should note that only few organic materials has an increase of $\Gamma(T)$ near $T_{\mathrm{g}}$ been reported for acoustic phonons at gigahertz frequencies. In these cases the effect was ascribed to a change in "local" viscosity. ${ }^{54,55}$

The temperature dependence of the longitudinal sound velocity can also reveal information on the PS amorphous phase. In a recent report, Schweizer and co-workers describe the glass transition in terms of the coupling between short scale hopping and long-range collective elastic distortions. ${ }^{56}$ In this treatment, the glass transition temperature is proposed to be proportional to the cohesive force parameter $B$ which can be obtained from the temperature dependence of the bulk modulus, $K=M-4 G / 3$. Because of the much smaller (about 4 times) $G$ and the virtually $T$-independent Poisson's ratio (Figure S3), we can use $K \approx M\left(=\rho c_{\mathrm{L}}{ }^{2}\right)$. The variation of $c_{\mathrm{L}}(T)$ with temperature is given by $c_{\mathrm{L}}(T)=B / T-A$, where $B$ and $A$ denote the cohesive force and entropic packing parameter, respectively. Application of this equation to represent the sound velocity in Figure $7 \mathrm{a}$ reveals that $B$ assumes very similar values $\left(B \approx 10 \times 10^{5} \mathrm{~m} . \mathrm{K} / \mathrm{s}\right)$ above $T_{\mathrm{g}}$ but distinct values $\left((1.5-2) \times 10^{5} \mathrm{~m} . \mathrm{K} / \mathrm{s}\right.$ for type $\mathrm{I}$ and $2.7 \times$ $10^{5} \mathrm{~m} . \mathrm{K} / \mathrm{s}$ for type II films in Table S4) below $T_{\mathrm{g}}$. The respective values of the bulk PS $30 \mathrm{k}$ film are $10 \times 10^{5} \mathrm{~m} . \mathrm{K} / \mathrm{s}$ above and $1.4 \times 10^{5} \mathrm{~m} . \mathrm{K} / \mathrm{s}$ below $T_{\mathrm{g}}$, in spite of the very similar $T_{\mathrm{g}}$ values. Our results are thus consistent with the proposition that the value of $T_{\mathrm{g}}$ is not entirely determined by $B .{ }^{56}$ The values of $B$ and $A$ for the four systems are listed in Table S4. In an alternative linear representation of $c(T)$, the slopes $\alpha_{\mathrm{g}} \equiv \mathrm{d} \ln$

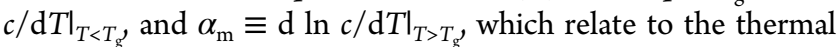
volume expansion below and above $T_{\mathrm{g}}$, as well as the slopes $\alpha_{\mathrm{g}}$ $\equiv \mathrm{d} \ln f /\left.\mathrm{d} T\right|_{T<T_{\mathrm{g}}}$ and $\alpha_{\mathrm{m}} \equiv \mathrm{d} \ln f /\left.\mathrm{d} T\right|_{T>T_{\mathrm{g}}}$ for the neat polymergrafted particles (Figure 4 and Figure S2) are listed in Table S3. For practical aspects of the particle brush materials, the effective medium sound velocity (Figure 7a) yields the elastic longitudinal modulus, $M$, at different temperatures. At $295 \mathrm{~K}$, the longitudinal modulus $M$ increases from $5.8 \mathrm{GPa}$ (bulk PS, $\left.\phi_{\mathrm{PS}}=1\right)$ to $13.1 \mathrm{GPa}\left(\mathrm{DP} 1170 \phi_{\mathrm{PS}}=0.5\right)$ assuming a linear volume fraction dependence for the mass density and $1050 \mathrm{~kg} /$ $\mathrm{m}^{3}$ for bulk PS and $1850 \mathrm{~kg} / \mathrm{m}^{3}$ for the silica core. ${ }^{26}$ At first glance, longitudinal sound velocity $c$ (Figure 7a) and hence $M$ increase with decreasing $\phi_{\mathrm{PS}}$ (from DP1300 to DP1170) due to the increase of the silica composition. However, a careful inspection reveals that $\phi_{\mathrm{PS}}$ is not the only parameter affecting $c$ as DP530 and DP100 ( $c=2710 \mathrm{~m} / \mathrm{s}$ at room temperature) exhibit a lower value than in DP1170 notably with similar $\phi_{\mathrm{PS}}$. 


\section{CONCLUSIONS}

Brillouin light scattering analysis of polymer-tethered particles in both pristine and film state reveals a distinctive dependence of particle-particle interactions and structure relaxation on the grafting density of brush particles. For individual brush particles (that are obtained from precipitation out of dilute solution) the interpretation of vibration eigenmode spectrum confirms more pronounced clustering and "brush fusion" in the case of sparse particle systems. This is rationalized as a consequence of the more relaxed chain conformation and more extensive chain entanglement in sparsely tethered systems. These structural characteristics imply that the fundamental $(1,2)$ and the interaction induced $(1,1)$ vibration modes prevail at temperatures above $T_{\mathrm{g}}$ only for these systems. In the film state, the glass transition is probed via the temperature dependence of the sound velocity as well as the lifetime of hypersonic phonons. While the first probes the glass transition via the material's thermal expansion (and is thus similar to a calorimetric measurement), the phonon lifetime depends on changes in the local viscosity which is sensitive to segment density distribution (and hence on the brush architecture). Analysis of the glass formation in the solid film state, accomplished by determination of the long-wavelength effective medium sound velocity, is found to not sensitively depend on grafting density. This is in support of previous studies that reported no measurable effect of the grafting density on the glass transition temperature of particle brush solids. In contrast, local (segmental) relaxation (tested via the hypersonic phonon lifetime) reveals a distinctive dependence on the grafting density. The phonon lifetime displays a sharp transition at $T_{\mathrm{g}}$ in the case of sparse brush systems (similar to linear polymers), whereas only a weak gradual transition is observed in densely tethered systems. Association to viscosity and hence to global dynamics is possible.

Our results provide (to the authors' knowledge) the first experimental evidence for the effect of particle brush architecture on the relaxation behavior in particle brush-based materials. Because the effect is primarily on the local viscosity, the effect is not detected in dilatometric experiments (such as sound velocity or calorimetric measurements) but rather becomes visible in the analysis of the phonon lifetime. The ability to track dynamical changes directly on the local and indirectly on more global scale renders Brillouin light scattering a versatile technique to probe glass transition processes in brush particle media. Given the critical importance of glass formation to practical aspects of materials in applications such as "aging behavior", it is hoped that the results will contribute to the development of more efficient methods for designing particle brush materials with tailored properties.

\section{EXPERIMENTAL SECTION}

Synthesis. Particle brush synthesis was performed using surfaceinitiated atom transfer radical polymerization following a procedure described previously. ${ }^{57}$ The final molar ratios of reaction components in a typical reaction were approximately $[\text { styrene }]_{0}:\left[\mathrm{SiO}_{2}-\mathrm{Br}\right]_{0}$ : $[\mathrm{CuBr}]_{0}:\left[\mathrm{CuBr}_{2}\right]_{0}:[\mathrm{PMDETA}]_{0}$ of 2000:1:2.5:0.25:2.75 with a volume fraction of nonreactive solvents of $5.4 \%$ dimethylformamide and $40 \%$ anisole in a $100 \mathrm{~mL}$ flask and stirred at approximately $1000 \mathrm{rpm}$. The polymerization was stopped by exposing the catalyst to oxygenated tetrahydrofuran after cooling under continuous stirring. The final product was dialyzed against tetrahydrofuran and methanol until the copper(II) catalyst was removed as evidenced by disappearance of its characteristic color.
Styrene (St, Aldrich, 99\%) was purified by passing through a basic alumina column before use. Copper(I) bromide was prepared by reduction of an aqueous solution of $\mathrm{CuBr}_{2}$ with an aqueous solution of ascorbic acid, and copper(I) chloride was prepared by reduction of $\mathrm{CuCl}_{2}$ aqueous solution using an aqueous solution of sodium sulfite. Both copper(I) halides were then sequentially filtered, washed with methanol, dried, and stored under vacuum before use. Silica nanoparticles $\left(\mathrm{SiO}_{2} \mathrm{NP}\right), 30 \%$ solution in isopropanol, effective diameter, $d_{\text {TEM }} \approx 113 \mathrm{~nm}$, were donated by Nissan Chemical Corporation and used as received. 5-Hexen-1-ol (98\%), $\alpha$ bromoisobutyryl bromide (98\%), triethoxysilane (95\%), ethyl 2bromoisobutyrate (EBiB, 98\%), 4,4'-dinonyl-2,2'-bipyridine (dNbpy, $99 \%), N, N, N^{\prime}, N^{\prime \prime}, N^{\prime \prime}$-pentamethyldiethylenetriamine (PMDETA, 99\%), and anisole (99\%) were purchased from Aldrich and used as received. All other chemicals and solvents were supplied by Aldrich and Acros Organics.

Particle brush films of $100 \mu \mathrm{m}$ thickness were prepared by casting of appropriate amounts of particle brush solution ( $4 \mathrm{wt} \%$ in toluene) on silicon substrates (with natural oxides on the surface) under ambient atmosphere. Films were dried and lifted off from substrate to allow for Brillouin light scattering from free-standing films. Under these conditions, compressive forces that arise during solvent evaporation are expected to result in colloidal crystal type microstructures. ${ }^{58}$

Size Exclusion Chromatography (SEC). Molecular weight and dispersity were measured by size exclusion chromatography using a Waters 515 pump and a Waters 2414 differential refractometer and PSS columns (Styrogel $10^{5}, 10^{3}$, and $10^{2} \AA$ ) in THF as an eluent (35 ${ }^{\circ} \mathrm{C}$, flow rate of $1 \mathrm{~mL} / \mathrm{min}$ ) with toluene and diphenyl ether as internal references. A linear polystyrene (PS) standard was used for calibration. To perform SEC, chains were cleaved from particles by etching of particles in HF in a polypropylene vial for $20 \mathrm{~h}$, neutralized with ammonium hydroxide, and dried with magnesium sulfate before running SEC. Hydrofluoric acid (50 vol \% HF) was purchased from Acros Organics and used as received. THF was purchased from Aldrich and used as received.

Thermogravimetric Analysis (TGA). Assessment of the grafting density and inorganic content of particles was made using weight fractions measured from thermogravimetric analysis (TGA) on a Q50 TGA analyzer from TA Instruments under nitrogen up to $850{ }^{\circ} \mathrm{C}$. Grafting density was calculated by using the weight fractions measured with TGA to convert to number of polymer chains using the molar mass of polymer chains (as determined using size exclusion chromatography) and surface area (using a silica mass density of $1.85 \mathrm{~g} / \mathrm{cm}^{3}$ ). The silica core was the same for all four particle brushes with radius $R_{\mathrm{c}}=57 \pm 3 \mathrm{~nm}$ as determined by TEM. The particle shape and size in the dry state were inferred from SEM images (Supporting Information), indicating spherical PS shell and revealing both molecular weight and grafting density thickness dependence. In addition, an estimate of the PS height, $h$, was obtained from the centerto center distance $d=2\left(R_{c}+h\right)$ in the TEM images of the assembled particle brush films. ${ }^{26}$ Given the different thermodynamic conditions, bad (air) and theta (film) solvent conditions, the two estimates of $h$ can be different.

Differential Scanning Calorimetry (DSC). Thermal characterization was performed using a DSC-Q20 (TA Instruments) with a heat rate of $10 \mathrm{~K} / \mathrm{min}$ and cooling rate of $20 \mathrm{~K} / \mathrm{min}$ across a temperature range of $293 \mathrm{~K}<T<403 \mathrm{~K}$. Three cycles of heating and cooling were performed to evaluate the effect of thermal treatment on the glass transition.

Brillouin Light Scattering (BLS). BLS measurements are performed by scattering of a probe laser beam from thermal excited phonons along $\mathbf{q}=\mathbf{k}_{\mathrm{s}}-\mathbf{k}_{\mathbf{i}}$, where $\mathbf{k}_{\mathbf{i}}$ and $\mathbf{k}_{\mathrm{s}}$ refer to incident and scattered photon vector, respectively. The resultant frequency shift at hypersonic (gigahertz) region is resolved by a high-resolution tandem Fabry-Pérot interferometer (JRS Instruments). The scattering vector q, equal to phonon wave vector, is independent of the refractive index in transmission geometry and characterizes the in-plane phonon propagations, whose magnitude is $q_{\|}=\sin (\theta / 2) 4 \pi / \lambda$, with $\theta$ and $\lambda=$ $532 \mathrm{~nm}$ being scattering angle and wavelength of incident light, respectively. By turning the polarization of scattering light, the 
longitudinal (vv polarization) and transverse (vh depolarization) waves are selected with input polarizer v. For the polymer brush films, the Brillouin frequency was $q$-dependent with a linear relation $\left(f=c_{i} q / 2 \pi\right.$, where $c_{i}$ is the longitudinal $\left(c_{\mathrm{L}}\right)$ sound velocity at $\mathrm{vv}$ and the transverse $\left(c_{\mathrm{T}}\right)$ sound velocity at vh polarization). For the polymer brush particles, the particle eigenmode spectra were recorded in the transmission geometry, which is $q$-independent due to the light multiple scattering. The sample temperature was monitored with platinum resistance temperature detector and controlled with a homemade temperature controller. For the temperature scan measurements, the sample was allowed to isothermally equilibrate for $20 \mathrm{~min}$ before the recording of spectra.

Theoretical Calculations. The elastic eigenmodes of a solid, uniform or core-shell, spherical particle, consisting respectively of one or two homogeneous and isotropic materials, and embedded in a fluid host matrix, are calculated through a multipole expansion of the elastic field inside and outside the particle into a basis of vector spherical waves. These elastic eigenmodes, characterized by the number $n$ denoting their order of appearance with increasing frequency (it coincides the number of radial nodes in the case of a homogeneous particle), the angular momentum $l$, the azimuthal number $m$, and the polarization $P$ (longitudinal $L$, and transverse, $M$ or $N$ ), are organized in two independent (uncoupled) subgroups: the torsional modes $(M$ polarized) and the spheroidal modes ( $L$ and $N$ polarized). Perfect boundary conditions (PBC), i.e., continuity of the normal and tangential components of the elastic displacement field and surface traction at any interface separating two different elastic media, are applied, thus leading to knowledge of the elastic field everywhere in space. The scattered field by such uniform or multishell spherical particles can be described with the help of a scattering transition $\mathbf{T}$ matrix formalism (explicit expressions can be found in ref 32) which, if combined with a Green's function formalism, ${ }^{31}$ allows for the calculation of the frequency position $f_{n l m}$ of the several virtual bound eigenstates of the elastic field, localized in the particle, through the change in the density of states (DOS) of the elastic field, induced by the presence of the sphere with respect to the infinite host matrix. We note that for spherically symmetric particles, as is the case here, these resonant modes are independent of $m$ (i.e., they are $(2 l+1)$ degenerated). Thus, we will denote their resonant frequency as $f_{n l}$, and for the first nodal mode $(n=1)$, we will omit for the sake of simplicity the index $n$.

\section{ASSOCIATED CONTENT}

\section{(S Supporting Information}

The Supporting Information is available free of charge on the ACS Publications website at DOI: 10.1021/acs.macromol.7b01752.

Images of particle brush in the isolated particle (SEM) and assembly film (TEM), temperature-dependent eigenmode spectra and DSC traces of particles, thermomechanical properties of particle brushes, and BLS spectra of PS (PDF)

\section{AUTHOR INFORMATION}

\section{Corresponding Authors}

*E-mail: bockstaller@cmu.edu (M.R.B.).

*E-mail: fytas@mpip-mainz.mpg.de (G.F.).

\section{ORCID}

Jiajun Yan: 0000-0003-3286-3268

Krzysztof Matyjaszewski: 0000-0003-1960-3402

Michael R. Bockstaller: 0000-0001-9046-9539

\section{Notes}

The authors declare no competing financial interest.

\section{ACKNOWLEDGMENTS}

M.B. and K.M. acknowledge financial support by the National Science Foundation (via Grants CMMI 1663305 and DMR 1501324) as well as the Scott Institute for Energy Technologies at Carnegie Mellon University. J.Z. acknowledges support by the Chinese Science Foundation. The work was supported by ERC AdG SmartPhon No. 694977.

\section{REFERENCES}

(1) Schmitt, M.; Zhang, J.; Lee, J.; Lee, B.; Ning, X.; Zhang, R.; Karim, A.; Davis, R. F.; Matyjaszewski, K.; Bockstaller, M. R. Polymer ligand-induced autonomous sorting and reversible phase separation in binary particle blends. Science Advances 2016, 2 (12), e1601484.

(2) Schmitt, M.; Choi, J.; Hui, C. M.; Chen, B.; Korkmaz, E.; Yan, J.; Margel, S.; Ozdoganlar, O. B.; Matyjaszewski, K.; Bockstaller, M. R. Processing fragile matter: effect of polymer graft modification on the mechanical properties and processibility of (nano-) particulate solids. Soft Matter 2016, 12 (15), 3527-3537.

(3) Alonso-Redondo, E.; Schmitt, M.; Urbach, Z.; Hui, C. M.; Sainidou, R.; Rembert, P.; Matyjaszewski, K.; Bockstaller, M. R.; Fytas, G. A new class of tunable hypersonic phononic crystals based on polymer-tethered colloids. Nat. Commun. 2015, 6, 8309.

(4) Grabowski, C. A.; Koerner, H.; Meth, J. S.; Dang, A.; Hui, C. M.; Matyjaszewski, K.; Bockstaller, M. R.; Durstock, M. F.; Vaia, R. A. Performance of dielectric nanocomposites: matrix-free, hairy nanoparticle assemblies and amorphous polymer-nanoparticle blends. ACS Appl. Mater. Interfaces 2014, 6 (23), 21500-9.

(5) Tchoul, M. N.; Fillery, S. P.; Koerner, H.; Drummy, L. F.; Oyerokun, F. T.; Mirau, P. A.; Durstock, M. F.; Vaia, R. A. Assemblies of titanium dioxide-polystyrene hybrid nanoparticles for dielectric applications. Chem. Mater. 2010, 22 (5), 1749-1759.

(6) Tao, P.; Viswanath, A.; Li, Y.; Rungta, A.; Benicewicz, B. C.; Siegel, R. W.; Schadler, L. S. Refractive Index Engineering of Polymer Nanocomposites Prepared by End-grafted Polymer Chains onto Inorganic Nanoparticles. MRS Online Proc. Libr. 2011, 1359, doi 10.1557/opl.2011.766.

(7) Pryamtisyn, V.; Ganesan, V.; Panagiotopoulos, A. Z.; Liu, H. J.; Kumar, S. K. Modeling the anisotropic self-assembly of spherical polymer-grafted nanoparticles. J. Chem. Phys. 2009, 131 (22), 221102.

(8) Li, Y.; Wang, L.; Natarajan, B.; Tao, P.; Benicewicz, B. C.; Ullal, C.; Schadler, L. S. Bimodal "matrix-free" polymer nanocomposites. RSC Adv. 2015, 5 (19), 14788-14795.

(9) Cangialosi, D.; Alegría, A.; Colmenero, J. Effect of nanostructure on the thermal glass transition and physical aging in polymer materials. Prog. Polym. Sci. 2016, 54-55, 128-147.

(10) Berthier, L.; Ediger, M. D. Facets of Glass Physics. Phys. Today 2016, 69 (1), 40-46.

(11) Lan, T.; Torkelson, J. M. Fragility-confinement effects: apparent universality as a function of scaled thickness in films of freely deposited, linear polymer and its absence in densely grafted brushes. Macromolecules 2016, 49 (4), 1331-1343.

(12) Qin, X.; Xia, W. J.; Sinko, R.; Keten, S. Tuning Glass Transition in Polymer Nanocomposites with Functionalized Cellulose Nanocrystals through Nanoconfinement. Nano Lett. 2015, 15 (10), 67386744.

(13) Oh, H.; Green, P. F. Polymer chain dynamics and glass transition in athermal polymer/nanoparticle mixtures. Nat. Mater. 2009, 8 (2), 139 .

(14) Bansal, A.; Yang, H. C.; Li, C. Z.; Cho, K. W.; Benicewicz, B. C.; Kumar, S. K.; Schadler, L. S. Quantitative equivalence between polymer nanocomposites and thin polymer films. Nat. Mater. 2005, 4 (9), 693-698.

(15) Akcora, P.; Liu, H.; Kumar, S. K.; Moll, J.; Li, Y.; Benicewicz, B. C.; Schadler, L. S.; Acehan, D.; Panagiotopoulos, A. Z.; Pryamitsyn, V.; Ganesan, V.; Ilavsky, J.; Thiyagarajan, P.; Colby, R. H.; Douglas, J. F. Anisotropic self-assembly of spherical polymer-grafted nanoparticles. Nat. Mater. 2009, 8 (4), 354-9. 
(16) Varol, H. S.; Meng, F.; Hosseinkhani, B.; Malm, C.; Bonn, D.; Bonn, M.; Zaccone, A.; Parekh, S. H. Nanoparticle amount, and not size, determines chain alignment and nonlinear hardening in polymer nanocomposites. Proc. Natl. Acad. Sci. U. S. A. 2017, 114 (16), E3170E3177.

(17) Koerner, H.; Opsitnick, E.; Grabowski, C. A.; Drummy, L. F.; Hsiao, M. S.; Che, J.; Pike, M.; Person, V.; Bockstaller, M. R.; Meth, J. S.; Vaia, R. A. Physical Aging and Glass Transition of Hairy Nanoparticle Assemblies. J. Polym. Sci., Part B: Polym. Phys. 2016, 54 (2), 319-330.

(18) Ellison, C. J.; Torkelson, J. M. The distribution of glasstransition temperatures in nanoscopically confined glass formers. Nat. Mater. 2003, 2 (10), 695-700.

(19) Chai, Y.; Salez, T.; McGraw, J. D.; Benzaquen, M.; DalnokiVeress, K.; Raphaël, E.; Forrest, J. A. A Direct Quantitative Measure of Surface Mobility in a Glassy Polymer. Science 2014, 343 (6174), 994999.

(20) Mark, C.; Holderer, O.; Allgaier, J.; Hübner, E.; PyckhoutHintzen, W.; Zamponi, M.; Radulescu, A.; Feoktystov, A.; Monkenbusch, M.; Jalarvo, N.; Richter, D. Polymer Chain Conformation and Dynamical Confinement in a Model OneComponent Nanocomposite. Phys. Rev. Lett. 2017, 119 (4), 047801.

(21) Alonso-Redondo, E.; Gueddida, A.; Huesmann, H.; El Abouti, O.; Tremel, W.; El Boudouti, E. H.; Djafari-Rouhani, B.; Fytas, G. Direction-dependent elastic properties and phononic behavior of PMMA/BaTiO 3 nanocomposite thin films. J. Chem. Phys. 2017, 146 (20), 203325.

(22) Still, T.; Mattarelli, M.; Kiefer, D.; Fytas, G.; Montagna, M. Eigenvibrations of Submicrometer Colloidal Spheres. J. Phys. Chem. Lett. 2010, 1 (16), 2440-2444.

(23) Kuok, M. H.; Lim, H. S.; Ng, S. C.; Liu, N. N.; Wang, Z. K. Brillouin study of the quantization of acoustic modes in nanospheres. Phys. Rev. Lett. 2003, 90 (25), 255502.

(24) Crut, A.; Maioli, P.; Del Fatti, N.; Vallee, F. Acoustic vibrations of metal nano-objects: Time-domain investigations. Phys. Rep. 2015, $549,1-43$.

(25) Girard, A.; Gehan, H.; Crut, A.; Mermet, A.; Saviot, L.; Margueritat, J. Mechanical Coupling in Gold Nanoparticles Supermolecules Revealed by Plasmon-Enhanced Ultralow Frequency Raman Spectroscopy. Nano Lett. 2016, 16 (6), 3843-9.

(26) Schneider, D.; Schmitt, M.; Hui, C. M.; Sainidou, R.; Rembert, P.; Matyjaszewski, K.; Bockstaller, M. R.; Fytas, G. Role of Polymer Graft Architecture on the Acoustic Eigenmode Formation in Densely Polymer-Tethered Colloidal Particles. ACS Macro Lett. 2014, 3 (10), 1059-1063.

(27) Portales, H.; Goubet, N.; Saviot, L.; Adichtchev, S.; Murray, D. B.; Mermet, A.; Duval, E.; Pileni, M. P. Probing atomic ordering and multiple twinning in metal nanocrystals through their vibrations. Proc. Natl. Acad. Sci. U. S. A. 2008, 105 (39), 14784-9.

(28) Mattarelli, M.; Montagna, M.; Still, T.; Schneider, D.; Fytas, G. Vibration spectroscopy of weakly interacting mesoscopic colloids. Soft Matter 2012, 8 (15), 4235-4243.

(29) Saviot, L.; Le Gallet, S.; Demoisson, F.; David, L.; Sudre, G.; Girard, A.; Margueritat, J.; Mermet, A. Inelastic Light Scattering Contribution to the Study of the Onset of Sintering of a Nanopowder. J. Phys. Chem. C 2017, 121 (4), 2487-2494.

(30) Kumar, S. K.; Jouault, N.; Benicewicz, B.; Neely, T. Nanocomposites with polymer grafted nanoparticles. Macromolecules 2013, 46 (9), 3199-3214.

(31) Sainidou, R.; Stefanou, N.; Modinos, A. Green's function formalism for phononic crystals. Phys. Rev. B: Condens. Matter Mater. Phys. 2004, 69 (6), 064301.

(32) Sainidou, R.; Stefanou, N.; Psarobas, I.; Modinos, A. A layermultiple-scattering method for phononic crystals and heterostructures of such. Comput. Phys. Commun. 2005, 166 (3), 197-240.

(33) Alonso-Redondo, E.; Gueddida, A.; Li, J.; Graczykowski, B.; Torres, C. M. S.; Pennec, Y.; Yang, S.; Djafari-Rouhani, B.; Fytas, G. Directional elastic wave propagation in high-aspect-ratio photoresist gratings: liquid infiltration and aging. Nanoscale 2017, 9 (8), 27392747.

(34) Lamb, H. On the Vibrations of an Elastic Sphere. Proceedings of the London Mathematical Society 1881, s1-13 (1), 189-212.

(35) Kim, S. A.; Mangal, R.; Archer, L. A. Relaxation Dynamics of Nanoparticle-Tethered Polymer Chains. Macromolecules 2015, 48 (17), 6280-6293.

(36) Borisov, O. V.; Zhulina, E. B.; Polotsky, A. A.; Leermakers, F. A. M.; Birshtein, T. M. Interactions between Brushes of Root-Tethered Dendrons. Macromolecules 2014, 47 (19), 6932-6945.

(37) Shin, K.; Obukhov, S.; Chen, J.-T.; Huh, J.; Hwang, Y.; Mok, S.; Dobriyal, P.; Thiyagarajan, P.; Russell, T. P. Enhanced mobility of confined polymers. Nat. Mater. 2007, 6 (12), 961-965.

(38) Cheng, W.; Sainidou, R.; Burgardt, P.; Stefanou, N.; Kiyanova, A.; Efremov, M.; Fytas, G.; Nealey, P. F. Elastic properties and glass transition of supported polymer thin films. Macromolecules 2007, 40 (20), 7283-7290.

(39) Friedman, E.; Ritger, A.; Andrews, R. Brillouin Scattering Near the Glass Transition of Polymethyl Methacrylate. J. Appl. Phys. 1969, 40 (11), 4243-4247.

(40) Mitchell, R. S.; Guillet, J. E. Brillouin-Scattering in Amorphous Polymeric Solids. J. Polym. Sci., Polym. Phys. Ed. 1974, 12 (4), 713733.

(41) Kearns, K. L.; Still, T.; Fytas, G.; Ediger, M. D. High-modulus organic glasses prepared by physical vapor deposition. Adv. Mater. 2010, 22 (1), 39-42.

(42) Brandrup, J.; Immergut, E. H.; Grulke, E. A.; Abe, A.; Bloch, D. R. Polymer Handbook; Wiley: New York, 1989; Vol. 7.

(43) Feng, S.; Li, Z.; Liu, R.; Mai, B.; Wu, Q.; Liang, G.; Gao, H.; $\mathrm{Zhu}, \mathrm{F}$. Glass transition of polystyrene nanospheres under different confined environments in aqueous dispersions. Soft Matter 2013, 9 (18), 4614-4620.

(44) Zhang, C.; Guo, Y.; Priestley, R. D. Glass Transition Temperature of Polymer Nanoparticles under Soft and Hard Confinement. Macromolecules 2011, 44 (10), 4001-4006.

(45) Sasaki, T.; Shimizu, A.; Mourey, T. H.; Thurau, C. T.; Ediger, M. Glass transition of small polystyrene spheres in aqueous suspensions. J. Chem. Phys. 2003, 119 (16), 8730-8735.

(46) Askar, S.; Li, L.; Torkelson, J. M. Polystyrene-Grafted Silica Nanoparticles: Investigating the Molecular Weight Dependence of Glass Transition and Fragility Behavior. Macromolecules 2017, 50 (4), 1589-1598.

(47) Agarwal, P.; Kim, S. A.; Archer, L. A. Crowded, Confined, and Frustrated: Dynamics of Molecules Tethered to Nanoparticles. Phys. Rev. Lett. 2012, 109 (25), 258301.

(48) Zuo, B.; Zhang, S.; Niu, C.; Zhou, H.; Sun, S.; Wang, X. Grafting density dominant glass transition of dry polystyrene brushes. Soft Matter 2017, 13 (13), 2426-2436.

(49) Yamamoto, S.; Tsujii, Y.; Fukuda, T. Glass Transition Temperatures of High-Density Poly(methyl methacrylate) Brushes. Macromolecules 2002, 35 (16), 6077-6079.

(50) Zhang, C.; Guo, Y. L.; Priestley, R. D. Glass Transition Temperature of Polymer Nanoparticles under Soft and Hard Confinement. Macromolecules 2011, 44 (10), 4001-4006.

(51) Chung, P. C.; Glynos, E.; Sakellariou, G.; Green, P. F. Elastic Mechanical Response of Thin Supported Star-Shaped Polymer Films. ACS Macro Lett. 2016, 5 (4), 439-443.

(52) Baeza, G. P.; Dessi, C.; Costanzo, S.; Zhao, D.; Gong, S.; Alegria, A.; Colby, R. H.; Rubinstein, M.; Vlassopoulos, D.; Kumar, S. K. Network dynamics in nanofilled polymers. Nat. Commun. 2016, 7, 11368 .

(53) Berne, B. J.; Pecora, R. Dynamic Light Scattering: With Applications to Chemistry, Biology, and Physics; Courier Corporation: 2013.

(54) Hosea, T. J. C.; Ng, S. C.; Oates, C. G. A Brillouin scattering study of the glass transition in sucrose. Food Hydrocolloids 1990, 4 (2), 137-147. 
(55) Kim, T. H.; Ko, J. H.; Kwon, E. M.; Jun, J. G. Micro-Brillouin Spectroscopy Applied to the Glass Transition of Anti-inflammatory Egonol. J. Opt. Soc. Korea 2010, 14 (4), 403-408.

(56) Mirigian, S.; Schweizer, K. S. Elastically cooperative activated barrier hopping theory of relaxation in viscous fluids. II. Thermal liquids. J. Chem. Phys. 2014, 140 (19), 194507.

(57) Hui, C. M.; Pietrasik, J.; Schmitt, M.; Mahoney, C.; Choi, J.; Bockstaller, M. R.; Matyjaszewski, K. Surface-Initiated Polymerization as an Enabling Tool for Multifunctional (Nano-)Engineered Hybrid Materials. Chem. Mater. 2014, 26 (1), 745-762.

(58) von Freymann, G.; Kitaev, V.; Lotsch, B. V.; Ozin, G. A. Bottom-up assembly of photonic crystals. Chem. Soc. Rev. 2013, 42 (7), $2528-2554$. 